\title{
Prosopagnosia associated with brain metastasis near the inferior longitudinal fasciculus in the nondominant temporal lobe: illustrative case
}

\author{
Hannah K. Weiss, MD, ${ }^{1}$ Donato R. Pacione, MD, ${ }^{1}$ Steven Galetta, MD, ${ }^{2}$ and Douglas Kondziolka, MD ${ }^{1}$ \\ Departments of ${ }^{1}$ Neurosurgery and ${ }^{2}$ Neurology, NYU Langone Health, New York, New York
}

BACKGROUND Disruptions of the inferior longitudinal fasciculus (ILF) in the nondominant temporal lobe can lead to the rare but significant higher visual-processing disturbance of prosopagnosia. Here, the authors describe a 57-year-old right hand-dominant female with a large breast cancer brain metastasis in the right temporal lobe who underwent resection and subsequent Gamma Knife radiosurgery. She presented with difficulty with facial recognition, but following surgical intervention, the prosopagnosia became more profound.

OBSERVATIONS Even in nondominant cortex, significant deficits can arise when operating near higher visual-processing centers, including the ILF.

LESSONS This case highlights the utility of imaging-based tractography obtained from preoperative imaging for resective surgical planning even when operating in areas that do not involve what is traditionally considered elegant areas of the brain. To optimize neurological outcomes in metastatic tumor resection, awareness and diffusion tensor imaging of neighboring, displaced white matter tracts may prevent permanent deficits in higher visual processing.

https://thejns.org/doi/abs/10.3171/CASE21313

KEYWORDS prosopagnosia; inferior longitudinal fasciculus; brain metastasis; face recognition; tractography

The inferior longitudinal fasciculus (ILF) is a white matter association tract between the temporal and occipital lobes that has a significant role in visual processing and visual memory. The ILF primarily serves as a connection between the occipital extrastriatal cortex and the anterior temporal lobe. ${ }^{1}$ The emergence of advanced imaging modalities and tractography, in conjunction with white matter dissection, has further elucidated subcomponents of the ILF, identified in some recent studies as four main subfasiculi: dorsolateral-occipital, cuneal, fusiform, and lingual branches; however, variability among individuals exists, and there remains ongoing discussion on these subcomponents. ${ }^{1-3}$ Recent anatomical studies have also shown that the volume of the ILF tends to be lateralized to the right; however, it has also been reported to have stronger connectivity on the left. ${ }^{1,3}$

The ILF is closely associated with neighboring white matter tracts. The ILF courses inferior to the superior longitudinal fasciculus, inferior and lateral to the arcuate fasciculus, and medial to the vertical occipital fasciculus, as well as close to the uncinate fasciculus in the medial temporal pole. ${ }^{3}$ The ILF is also closely associated with primary visual pathways, coursing inferior and lateral to the optic radiations in the temporal lobe. ${ }^{4}$

Disruption to the ILF and other associated neighboring white matter tracts can lead to a significant variety of language and visual disturbances. Lesions of the anterior optic radiation in the temporal lobe, Meyers Loop, results in the characteristic superior homonymous quadrantanopia. ${ }^{5}$ Disruption of the arcuate fasciculus and sucperior longitudinal fasciculus, in the dominant hemisphere, can lead to disorders of language processing and dysarthria. ${ }^{6}$ Interruption of the uncinate fasciculus can cause semantic paraphasias. ${ }^{6}$

Prosopagnosia, the inability to recognize familiar faces, is a unique disorder that develops from a disruption of the ILF. ${ }^{7}$ Prosopagnosia is a rare condition but has been described in scattered case reports throughout the years, caused by a variety of etiologies, including progressive degeneration of the temporal lobes, vascular anomalies of the posterior cerebral arteries, congenital cases, and neoplastic lesions interfering with connections to the fusiform face area. $^{8-11}$ Generally, prosopagnosia presents as inability recognize

ABBREVIATIONS FLAIR = fluid-attenuated inversion recovery; ILF = inferior longitudinal fasciculus; MRI = magnetic resonance imaging. INCLUDE WHEN CITING Published September 6, 2021; DOI: 10.3171/CASE21313.

SUBMITTED May 20, 2021. ACCEPTED June 9, 2021.

(C) 2021 The authors, CC BY-NC-ND 4.0 (http://creativecommons.org/licenses/by-nc-nd/4.0/). 
any familiar faces, even in immediate family members, without deficits in other visual processes. ${ }^{12}$ Right hemisphere dominance has been demonstrated in the development of prosopagnosia. ${ }^{12-14}$ In this report, we describe a patient with acquired prosopagnosia that becomes more profound following a resection of a metastatic lesion near the ILF in the nondominant temporal lobe.

\section{Illustrative Case}

The patient is a right hand dominant 57-year-old female with locally advanced triple-negative breast cancer treated with bilateral mastectomy and axillary dissection, neoadjuvant chemotherapy (doxorubicin, paclitaxel, capecitabine), and chest wall radiation. At the time of presentation, 2 years after her initial diagnosis, she had no known evidence of ongoing disease. She presented to the emergency department with complaints of 10 days of progressive headaches, localized to her right side, with associated nausea, vomiting, and "flashing light" visual disturbances in the left hemifield. It was also noted that she had been having mild difficulty with facial recognition for 2 months. Her physical exam was significant for left homonymous superior quadrantanopia involving the entire quadrant.

Computed tomography brain imaging revealed a hypodense, multilobulated lesion in the right posterior temporal lobe with associated low-density edema and mass effect. On magnetic resonance imaging (MRI), the irregular mass was measured at $6.0 \times 3.8 \times 4.0 \mathrm{~cm}$ with thin peripheral enhancement increased at the inferior margin with surrounding T2/fluid-attenuated inversion recovery (FLAIR) signal abnormality extending superiorly along the temporal stem and along the internal capsule (Figs. 1 and 2).

A right temporal craniotomy for resection of the mass was performed 3 days after admission. The tumor was noted to be partly cystic. The pathology was consistent with metastatic carcinoma.

Postoperative MRI showed edema but no definite enhancement along the resection margins (Fig. 3). The FLAIR sequences demonstrated a disruption of the ILF at the posterior superior aspect of the resection cavity. She recovered well with a stable left homonymous

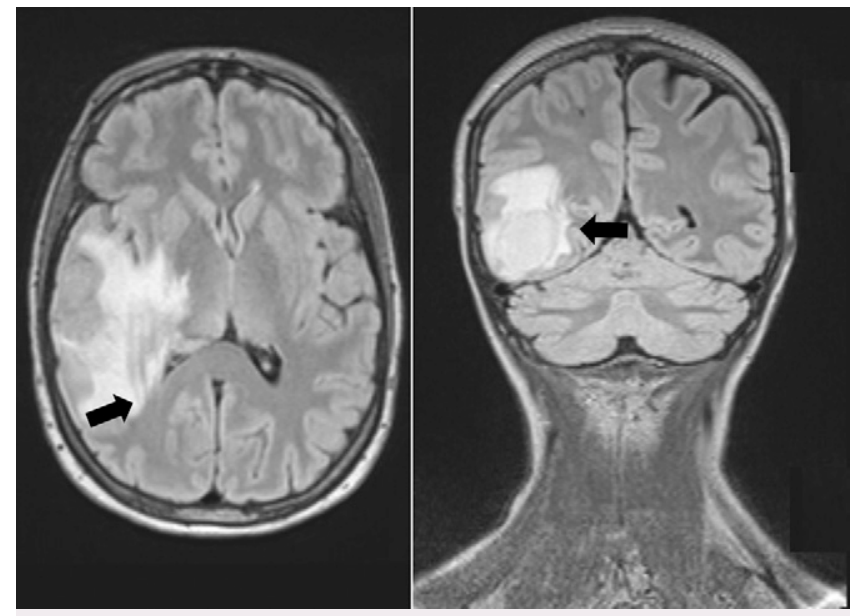

FIG. 1. MRI of metastatic breast adenocarcinoma in the right temporal lobe. Left: An axial T2-weighted FLAIR MRI scan. Right: A coronal T2-weighted FLAIR MRI scan, obtained upon presentation in the preoperative period. The surrounding T2/FLAIR signal abnormality extends superiorly and involves white matter tracts, including the typical location of the ILF (arrows).
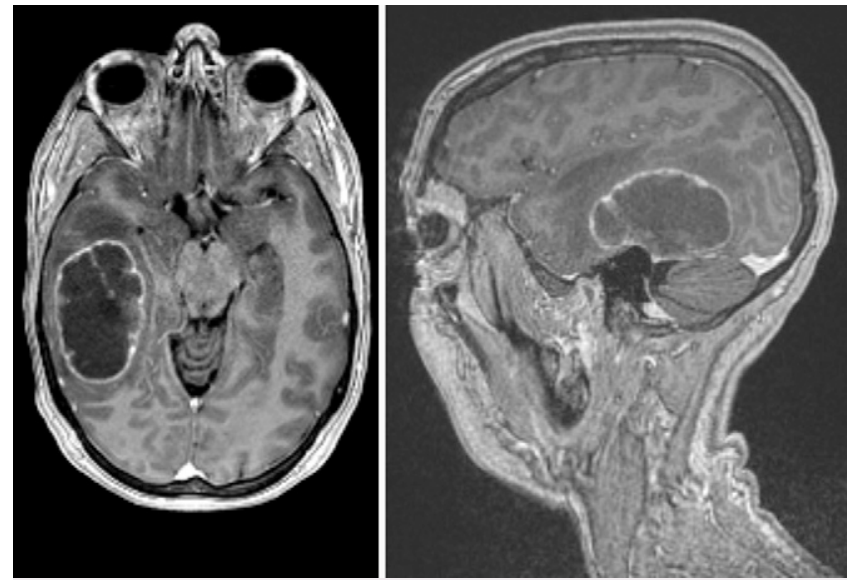

FIG. 2. MRI of metastatic breast adenocarcinoma in the right temporal lobe. Left: An axial postcontrast T1-weighted MRI scan. Right: sagittal multiplanar reconstruction MR image. There is nodular, thin peripheral enhancement surrounding the cystic metastatic lesion. These images were also obtained upon presentation, prior to resection.

superior quadrantanopia. After discharge from the hospital, she underwent local tumor bed Gamma Knife radiosurgery (8 Gy to the $50 \%$ isodose line at each of three sessions). After resection, she described having more profound prosopagnosia, occasionally even having difficulty recognizing the faces of her immediate family members (Video 1). She experienced occasional visual, gustatory, and auditory hallucinations as well.

VIDEO 1. Patient describing her experience with prosopagnosia.

Click here to view.

Approximately 3 years following radiosurgery, two additional small tumor nodules were found, and she underwent radiosurgery. Her prosopagnosia remained stable, and she also reported visual agnosia of new objects while driving. She was referred for formal visual-processing
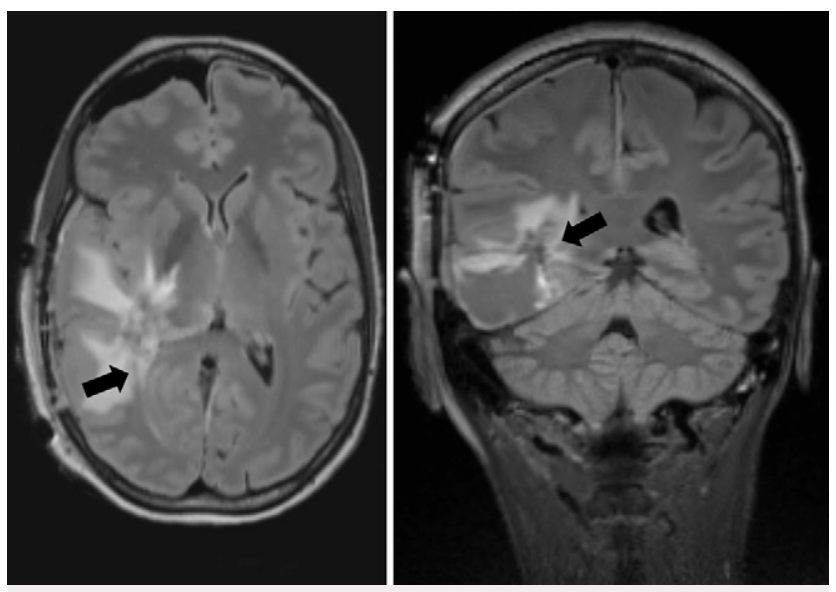

FIG. 3. MRI 1 day following craniotomy and resection. Left: An axial T2-weighted FLAIR MRI scan. Right: A coronal T2-weighted FLAIR MRI scan. There is continued edema throughout the right temporal lobe with T2/FLAIR signal abnormality surrounding white matter tracts near typical location of the ILF (arrows). 
testing, which revealed a score of $57 / 75$ on the Exposure Based Face Memory Test, which was considered a slightly above average score. She continues to endorse occasionally not recognizing her husband on the street or confusing another child for her daughter. She has no difficulty recognizing familiar cars, houses, or animals. She has adapted to these higher cortical visual deficits by using tactile, voice, and other specific mannerisms to identify familiar individuals.

\section{Discussion \\ Observations}

This case illustrates the rare complication of prosopagnosia arising from a lesion in the ILF in the nondominant temporal lobe. Prosopagnosia, first officially defined in 1947, has been noted in case reports and small case series throughout the last 70 years. ${ }^{15,16}$ The inability to recognize faces can have a significant impact on the psychosocial aspects of daily quality of life. ${ }^{17,18}$ Prosopagnosia most often occurs with bilateral lesions involving the ILF, but it has been occasionally noted with unilateral lesions of the right ILF that disconnect the visual areas from the facial recognition region in the anterior temporal lobe. ${ }^{12,13}$

\section{Lessons}

Even in the nondominant hemisphere, significant deficits can arise when operating near higher visual-processing centers, including the ILF. This case highlights the potential utility of imagingbased tractography obtained from preoperative imaging for surgical planning of tumor resections, even when operating in areas that do not involve what is traditionally considered critical areas. In the temporal lobe, disruption of the Meyers Loop visual fibers can lead to superior homonymous quadrantanopia, which tends to improve and have low impact on vision over time with spontaneous oculomotor adaptation. ${ }^{19-22}$ However, disruption of the ILF in the nondominant hemisphere can cause serious higher visual-processing disorders, having a more profound impact on psychosocial interactions. ${ }^{17,18}$ The addition of tractography to assist with intraoperative mapping of nondominant white matter tracts in the temporal lobe may have decreased the risk of this disturbance of higher visual processing. In particular, the extensive superior dissection of the metastatic lesion could have been avoided, possibly preventing the worsening prosopagnosia that developed postoperatively. However, this was a large and life-threatening tumor.

Brain metastasis, contrary to glial tumors, tend to displace rather than invade the white matter fiber tracts. ${ }^{23}$ The quality of diffusion tensor imaging and subsequent tractography is increased in metastatic lesions that displace rather than invade fibers, further emphasizing the utility of tractography during nondominant metastatic lesion resection. ${ }^{6,24}$ As most metastatic lesions will undergo postoperative irradiation to the resected tumor bed, the surgical extent of margins near white matter tracts can be altered to preserve higher visual processing and still maximize tumor control. ${ }^{25-27}$

Patients undergoing resection of metastatic tumors in the nondominant temporal lobe are at risk for developing critical, lifelong disturbances of higher visual processing in addition to language deficits. In order to reduce neurological complications and maintain adequate tumor resection margins, knowledge of the white matter tract anatomy in the occipitotemporal lobes as well as preoperative and intraoperative use of imaging-based tractography are of great importance. The possible neurological deficits arising from metastatic tumor resection near or within nondominant ILF should be discussed with the patient prior to the procedure and taken into consideration during preoperative and subsequent radiation therapy planning.

\section{References}

1. Panesar SS, Yeh F-C, Jacquesson T, Hula W, Fernandez-Miranda JC. A quantitative tractography study into the connectivity, segmentation and laterality of the human inferior longitudinal fasciculus. Front Neuroanat. 2018:12:47.

2. Catani M, Jones DK, Donato R, Ffytche DH. Occipito-temporal connections in the human brain. Brain. 2003;126(Pt 9):2093-2107.

3. Latini F, Mårtensson J, Larsson E-M, et al. Segmentation of the inferior longitudinal fasciculus in the human brain: a white matter dissection and diffusion tensor tractography study. Brain Res. 2017:1675:102-115.

4. Martin J. Neuroanatomy: Text and Atlas. 5th ed. McGraw Hill; 2021.

5. Powell HWR, Parker GJM, Alexander DC, et al. MR tractography predicts visual field defects following temporal lobe resection. Neurology. 2005;65(4):596-599.

6. Chang EF, Raygor KP, Berger MS. Contemporary model of language organization: an overview for neurosurgeons. J Neurosurg. 2015;122(2):250-261.

7. Herbet G, Zemmoura I, Duffau H. Functional anatomy of the inferior longitudinal fasciculus: from historical reports to current hypotheses. Front Neuroanat. 2018;12:77.

8. Cals N, Devuyst G, Afsar N, Karapanayiotides T, Bogousslavsky J. Pure superficial posterior cerebral artery territory infarction in The Lausanne Stroke Registry. J Neurol. 2002;249(7):855-861.

9. Joubert S, Felician O, Barbeau E, et al. Impaired configurational processing in a case of progressive prosopagnosia associated with predominant right temporal lobe atrophy. Brain. 2003;126(Pt 11): 2537-2550.

10. Pertzov Y, Krill D, Weiss N, Lesinger K, Avidan G. Rapid forgetting of faces in congenital prosopagnosia. Cortex. 2020;129:119-132.

11. Uttner I, Bliem H, Danek A. Prosopagnosia after unilateral right cerebral infarction. J Neurol. 2002;249(7):933-935.

12. Sorger B, Goebel R, Schiltz C, Rossion B. Understanding the functional neuroanatomy of acquired prosopagnosia. Neuroimage. 2007;35(2):836-852.

13. Meadows JC. The anatomical basis of prosopagnosia. J Neurol Neurosurg Psychiatry. 1974;37(5):489-501.

14. Sergent J, Signoret JL. Varieties of functional deficits in prosopagnosia. Cereb Cortex. 1992;2(5):375-388.

15. Bodamer J. [Prosop's agnosia; the agnosia of cognition]. Arch Psychiatr Nervenkr Z Gesamte Neurol Psychiatr. 1947;118(1-2):6-53.

16. Inoue S, Kondoh T, Nishihara M, Hosoda K, Kohmura E. [Transient prosopagnosia after removal of a tumor in the right occipito-temporal cortex: a case report]. No Shinkei Geka. 2008;36(11): 1023-1027.

17. Dalrymple KA, Fletcher K, Corrow S, et al. "A room full of strangers every day": the psychosocial impact of developmental prosopagnosia on children and their families. J Psychosom Res. 2014;77(2):144-150.

18. Yardley L, McDermott L, Pisarski S, Duchaine B, Nakayama K. Psychosocial consequences of developmental prosopagnosia: a problem of recognition. J Psychosom Res. 2008;65(5):445-451.

19. Schuett S, Kentridge RW, Zihl J, Heywood CA. Are hemianopic reading and visual exploration impairments visually elicited? New insights from eye movements in simulated hemianopia. Neuropsychologia. 2009;47(3):733-746.

20. Schuett S, Kentridge RW, Zihl J, Heywood CA. Adaptation of eyemovements to simulated hemianopia in reading and visual exploration: transfer or specificity? Neuropsychologia. 2009;47(7):1712-1720.

21. Zhang X, Kedar S, Lynn MJ, Newman NJ, Biousse V. Natural history of homonymous hemianopia. Neurology. 2006;66(6):901-905.

22. Zihl J. Eye movement patterns in hemianopic dyslexia. Brain. 1995;118(Pt 4):891-912. 
23. Liu X, Kinoshita M, Shinohara $H$, et al. Direct evidence of the relationship between brain metastatic adenocarcinoma and white matter fibers: a fiber dissection and diffusion tensor imaging tractography study. J Clin Neurosci. 2020;77:55-61.

24. Witwer BP, Moftakhar R, Hasan KM, et al. Diffusion-tensor imaging of white matter tracts in patients with cerebral neoplasm. $J$ Neurosurg. 2002;97(3):568-575.

25. Choi JW, Im YS, Kong DS, Seol HJ, Nam DH, Lee JI. Effectiveness of postoperative Gamma Knife radiosurgery to the tumor bed after resection of brain metastases. World Neurosurg. 2015;84(6): 1752-1757.

26. Quigley MR, Fuhrer R, Karlovits S, Karlovits B, Johnson M. Single session stereotactic radiosurgery boost to the post-operative site in lieu of whole brain radiation in metastatic brain disease. J Neurooncol. 2008;87(3):327-332.

27. Traylor JI, Habib A, Patel R, et al. Fractionated stereotactic radiotherapy for local control of resected brain metastases. J Neurooncol. 2019;144(2):343-350.

\section{Disclosures}

The authors report no conflict of interest concerning the materials or methods used in this study or the findings specified in this paper.

\section{Author Contributions}

Conception and design: Weiss, Pacione, Kondziolka. Acquisition of data: all authors. Analysis and interpretation of data: Weiss, Galetta, Kondziolka. Drafting the article: Weiss, Pacione, Kondziolka. Critically revising the article: Pacione, Kondziolka. Reviewed submitted version of manuscript: Weiss, Pacione.

\section{Supplemental Information \\ Video}

Video 1. https://vimeo.com/563253822

\section{Correspondence}

Hannah K. Weiss: NYU Langone Health, New York, NY. hannah. weiss@nyulangone.org. 